\title{
A matrix approach for stationary analysis fluid model driven by an $M / M / 1 / N$ queue with discouraged arrivals
}

\author{
Mahdy S. EL-paoumy ${ }^{1}$ and Taha Radwan ${ }^{2,3^{*}}$ (D)
}

\author{
*Correspondence: taha_ali_2003@ \\ hotmail.com \\ ${ }^{2}$ Department of Mathematics, \\ College of Sciences and Arts in \\ Ar-Rass, Qassim University, Ar-Rass, \\ Saudi Arabia \\ ${ }^{3}$ Department of Mathematics and \\ Statistics, Faculty of Management \\ Technology and Information \\ Systems, Port Said University, Port \\ Said, Egypt \\ Full list of author information is \\ available at the end of the article
}

\begin{abstract}
In this paper, a fluid queue driven by truncated queue with discouraged arrivals is considered. Using the efficient matrix technique, the expressions of the steady-state distribution of both the buffer content and stationary state probabilities of background birth-death process are acquired. Then, performance measures and analysis of server utilization and mean buffer content are carried out.
\end{abstract}

Keywords: Fluid queue, M/M/1/N queue, Discouraged arrivals, Buffer content, Matrix approach method

Mathematics Subject Classification (2000): 90B22, 60K25, 68M20, 44A10

\section{Introduction}

The study of fluid queueing system with finite space is very useful and important in a plethora of modern applications. In fact, there are numerous situations in which such phenomena occur and need to be investigated, for example, traffic shaping and modeling of transport control protocol, computer networks, and inventory and production systems, see also Adan [1], Anick et al. [2], Barbot [3], Kulkarni [4], and Mitra [5] for more details.

Many authors have studied fluid queues driven by a finite queueing system. Closed form expressions of eigenvalues and eigenvectors are obtained by Lenin and Parthasarathy [6] for the tridiagonal matrix in fluid queues driven by an $M / M / 1 / N$ queue. Further, the distribution of the exact buffer occupancy is obtained based on a spectral expansion. Lenin and Parthasarathy [7] considered an infinite capacity fluid buffer in which fluid at variable rates is received and released in a similar way to a state of truncated birth-death process. Mao et al. [8, 9] discussed a fluid model driven by a simple queue having single and multiple exponential vacations. Therefore, a system of first order homogeneous linear differential equations is derived for the distribution of the trivariate process of external environment and buffer content and is solved employing the standard spectral method. Viswanathan et al. [10] used two independent finite state birth-death processes to drive a fluid queue model in order to study the buffer occupancy distribution in high-speed networks. Furthermore, several studies have discussed the fluid queues driven by birth-death process such as [11-14].

(c) The Author(s). 2020 Open Access This article is distributed under the terms of the Creative Commons Attribution 4.0 International License (http://creativecommons.org/licenses/by/4.0/), which permits unrestricted use, distribution, and reproduction in any medium, provided you give appropriate credit to the original author(s) and the source, provide a link to the Creative Commons license, and indicate if changes were made. 
In this paper, the authors analyze a fluid model driven by a simple queue with discouraged arrivals. In particular, a system matrix for Laplace transform is derived for the steady-state distribution of the occupancy of buffer, in the "Model description" section, and solve it using the matrix approach method in the "Stationary solution of fluid queue driven by $M / M / 1 / N$ queue with discouraged arrivals" section. Some performance measures, such as mean buffer content and server utilization, are obtained in the "Some performance measures in fluid model" section. Finally, the numerical illustrations and conclusions are presented in the "Numerical illustrations" and "Conclusions" sections.

\section{Model description}

Assume that there is a fluid model driven by a single-server queueing process having state-dependent arrival and service rates. The model is structured from an infinitely large buffer where the fluid flow is regulated via the state of the background queueing process. Denote the background queuing process by $\{X(t), t \geq 0\}$ which has values in $\Omega=\{0,1,2, \ldots, N\}$, and let $p_{j}=\lim _{t \rightarrow \infty} P\{X(t)=j\}, j \in \Omega$. Here, $X(t)$ refers to the number of customers in the system at time $t$. Let $\lambda_{j}$ and $\mu_{j}$ denote the mean arrival and service rates, respectively, when there are $j$ customers in the queue. Both the interarrival times and the service times are exponentially distributed. In addition, the service discipline is first in first out (FIFO). We denote by $Z(t)$, the content of the buffer, i.e., the amount of fluid in the buffer, at time $t$. We will assume that the buffer content changes do not depend on $X$, i.e., the input rate minus the output rate, such that it can take both positive or negative values. For the case where the buffer is empty and the Markov Process is in a state 0 with rate $r_{0}<0$, therefore the buffer will be still empty. Assume $\mu_{0}=\lambda_{N}=0$ and $\mu_{j}=\lambda_{j}=0$, if $j \notin \Omega$. It is obvious that, the 2-dimensional process $\{X(t), Z(t), t \geq 0\}$ creates a Markov process with unique stationary distribution under a suitable stability condition [15].

Therefore, the following differential equation describes time change in $Z(t)$,

$$
\frac{d Z(t)}{d t}=\left\{\begin{array}{l}
0, \quad \text { if } Z(t)=0, \text { and } X(t)=0 \\
r_{0}, \text { if } Z(t)=0, \text { and } X(t)>0 \\
r, \text { if } Z(t)>0
\end{array}\right.
$$

The limit distribution for $Z(t)$ exists as $t \rightarrow \infty$, and the stationary net input rate must be negative [7], i.e.,

$$
d=r_{0} p_{0}+r \sum_{j=1}^{N} p_{j}<0
$$

where $p_{j}, j \in \Omega$ are the stationary state probabilities corresponding to the background birth-death process. Further, assume that the above stability conditions are satisfied.

Letting

$$
F_{j}(t, z) \equiv \operatorname{Pr}\{X(t)=j, Z(t) \leq z\}, j \in \Omega, \quad t, z \geq 0,
$$

The steady state of $F_{j}(t, z)$ can be obtained as $t \rightarrow \infty$ by the following formula: 


$$
F_{j}(z) \equiv \lim _{t \rightarrow \infty} \operatorname{Pr}\{X(t)=j, Z(t) \leq z\}, j \in \Omega, \quad z \geq 0
$$

It can be shown that the Kolmogorov forward equations for the Markov process $\{X(t), Z(t), t \geq 0\}$ are represented as

$$
\begin{aligned}
\frac{\partial F_{0}(t, z)}{\partial t}= & -r_{0} \frac{\partial F_{0}(t, z)}{\partial z}-\lambda_{0} F_{0}(t, z)+\mu_{1} F_{1}(t, z) \\
\frac{\partial F_{j}(t, z)}{\partial t}= & -r \frac{\partial F_{j}(t, z)}{\partial z}-\left(\lambda_{j}+\mu_{j}\right) F_{j}(t, z)+\lambda_{j-1} F_{j-1}(t, z) \\
& +\mu_{j+1} F_{j+1}(t, z), j \in \Omega-\{0, N\} \\
\frac{\partial F_{N}(t, z)}{\partial t}= & -r \frac{\partial F_{N}(t, z)}{\partial z}+\lambda_{N-1} F_{N-1}(t, z)-\mu_{N} F_{N}(t, z),
\end{aligned}
$$

Assume that the process is in equilibrium state $\partial F_{j}(t, z) / \partial t \equiv 0$ and $F_{j}(t, z) \equiv F_{j}(z)$. Hence, the above system (5-7) is reduced to the next system of ODEs:

$$
\begin{aligned}
& \frac{d F_{0}(z)}{d z}=-\frac{\lambda_{0}}{r_{0}} F_{0}(z)+\frac{\mu_{1}}{r_{0}} F_{1}(z), \\
& \frac{d F_{j}(z)}{d z}=-\frac{\left(\lambda_{j}+\mu_{j}\right)}{r} F_{j}(z)+\frac{\lambda_{j-1}}{r} F_{j-1}(z)+\frac{\mu_{j+1}}{r} F_{j+1}(z), z \geq 0, \quad j \in \Omega-\{0, N\} \\
& \frac{d F_{N}(z)}{d z}=\frac{\lambda_{N-1}}{r} F_{N-1}(z)-\frac{\mu_{N}}{r} F_{N}(z),
\end{aligned}
$$

The buffer content increases for positive the net input rate of fluid flow into the buffer such that the buffer cannot stay empty. It follows that the solution to (8-10) must satisfy the boundary conditions.

$$
\begin{aligned}
& F_{j}(0)=0, j \in \Omega, r>0 \\
& \operatorname{Pr}\{Z=0\}=F_{0}(0)=d_{0}, \text { for some constant } d_{0}\left(0<d_{0}<1\right)
\end{aligned}
$$

The stationary probability of the empty fluid queue is expressed as:

$$
\operatorname{Pr}\{Z=0\}=\frac{d}{r_{0}}=\frac{r_{0} p_{0}+\sum_{j=1}^{N} r p_{j}}{r_{0}}=\frac{r_{0} p_{0}+r\left(1-p_{0}\right)}{r_{0}} .
$$

Moreover, the following relation should also be satisfied

$$
F_{j}(\infty) \equiv \lim _{z \rightarrow \infty} F_{j}(z)=p_{j}, \quad j \in \Omega .
$$

\section{Stationary solution of fluid queue driven by $M / M / 1 / N$ queue with discouraged arrivals}

The fluid model discussed in the previous section is investigated when it has the background process as an $M / M / 1 / N$ queue with mean arrival and service rates to be $\lambda_{j}$ $=\frac{\lambda}{j+1}$ and $\mu_{j}=\mu$, respectively.

Taking Laplace transform of Eqs. (8-10), with respect to $z$, we have

$$
s F_{0}{ }^{*}(s)-F_{0}(0)=-\frac{\lambda_{0}}{r_{0}} F_{0}{ }^{*}(s)+\frac{\mu_{1}}{r_{0}} F^{*}{ }_{1}(s)
$$




$$
\begin{aligned}
s F_{j}^{*}(s)-F_{j}(0)= & -\frac{\left(\lambda_{j}+\mu_{j}\right)}{r_{j}} F_{j}^{*}(s)+\frac{\lambda_{j-1}}{r_{j}} F_{j-1}{ }^{*}(s) \\
& +\frac{\mu_{j+1}}{r_{j}} F_{j+1}{ }^{*}(s), s \geq 0, j \in \Omega-\{0, N\} \\
s F_{N}^{*}(s)-F_{N}(0)= & \frac{\lambda_{N-1}}{r_{N}} F_{N-1}{ }^{*}(s)-\frac{\mu_{N}}{r_{N}} F_{N}^{*}(s),
\end{aligned}
$$

where

$$
F_{j}^{*}(s)=\int_{0}^{\infty} e^{-s z} F_{j}(z) d z \text { and } F_{0}(0)=d_{0}
$$

Matrix notation (15-17) can be given as:

$$
A(s) \mathbb{F}^{*}(s)=\mathbb{F}(0)
$$

where $\mathbb{F}^{*}(s)=\left[F^{*}{ }_{0}(s), F^{*}{ }_{1}(s), \ldots, F^{*}{ }_{N}(s)\right]^{T}, \mathbb{F}(0)=\left[F_{0}(0), F_{1}(0), \ldots, F_{N}(0)\right]^{T}$ and

$$
A(s)=\left(\begin{array}{cccccc}
s+\frac{\lambda_{0}}{r_{0}} & -\frac{\mu_{1}}{r_{0}} & 0 \ldots & 0 & 0 \\
-\frac{\lambda_{0}}{r} & s+\frac{\lambda_{1}+\mu_{1}}{r} & -\frac{\mu_{2}}{r} \ldots & 0 & 0 \\
0 & -\frac{\lambda_{1}}{r} & s+\frac{\lambda_{2}+\mu_{2}}{r} & -\frac{\mu_{3}}{r} \ldots & 0 & 0 \\
\cdot & \cdot & . & . & 0 & 0 \\
\cdot & \cdot & \cdot & . & 0 & 0 \\
\cdot & \cdot & \cdot & . & 0 & 0 \\
0 & 0 & 0 & 0 \ldots & -\frac{\lambda_{N-1}}{r} & s+\frac{\mu_{N}}{r}
\end{array}\right)
$$

The matrix $A(s)$ transforms into a symmetric tridiagonal matrix by diagonal matrix

$$
\Phi=\operatorname{diag}\left[\phi_{0}, \phi_{1}, \ldots, \phi_{N}\right]
$$

with

$$
\phi_{0}=1, \quad \phi_{i}=\sqrt{\frac{i ! r}{r_{0}}\left(\frac{\mu}{\lambda}\right)^{i}} \quad, i=1,2, \ldots, N
$$

and we get

$$
s I+B=\Phi A \Phi^{-1}
$$

where the symmetric tridiagonal matrix $B$ with elements are the same that of matrix $A(s)$. 


$$
B=\left(\begin{array}{cccccc}
\frac{\lambda_{0}}{r_{0}} & \sqrt{\frac{\lambda \mu}{r_{0} r}} & 0 & 0 \ldots & 0 & 0 \\
\sqrt{\frac{\lambda \mu}{r_{0} r}} & \frac{\lambda}{2 r}+\frac{\mu}{r} & \sqrt{\frac{\lambda \mu}{2 r^{2}}} & 0 \ldots & 0 & 0 \\
0 & \sqrt{\frac{\lambda \mu}{2 r^{2}}} & \frac{\lambda}{3 r}+\frac{\mu}{r} & \sqrt{\frac{\lambda \mu}{3 r^{2}}} \ldots & 0 & 0 \\
\cdot & \cdot & \cdot & \cdot & 0 & 0 \\
\cdot & \cdot & \cdot & \cdot & 0 & 0 \\
0 & 0 & 0 & 0 \ldots & \frac{\lambda}{(N-1) r}+\frac{\mu}{r} & \sqrt{\frac{\lambda \mu}{N r^{2}}} \\
0 & 0 & 0 & 0 \ldots & \sqrt{\frac{\lambda \mu}{N r^{2}}} & \frac{\mu}{r}
\end{array}\right)
$$

If we take the determinant $\theta_{n}(s)$ of the bottom right square submatrices of the matrix $A(s)$ and $\beta_{n}(s)$ is the determinant of the top left submatrices of the matrix $A(s)$, then $\theta_{n}(s)$ and $\beta_{n}(s)$ satisfy the following difference equations.

$$
\begin{aligned}
& \theta_{n}(s)-\left(s+\left(\frac{\lambda}{N-n+2)}+\mu\right) \frac{1}{r}\right) \theta_{n-1}(s)+\frac{\lambda \mu}{r^{2}(N-n+2)} \theta_{n-2}(s)=0 \\
& \beta_{n}(s)-\left(s+\left(\frac{\lambda}{n}+\mu\right) \frac{1}{r}\right) \beta_{n-1}(s)+\frac{\lambda \mu}{(n-1) r^{2}} \beta_{n-2}(s)=0, \quad n=2,3, \ldots, N .
\end{aligned}
$$

with initial conditions

$$
\theta_{0}(s)=1=\beta_{0}(s) \text { and } \theta_{1}(s)=s+\frac{\mu}{r}, \beta_{1}(s)=s+\frac{\lambda}{r_{0}}
$$

Can be represent the elements of the inverse of the matrix $s I+B$ as following

$$
c_{i j}(s)= \begin{cases}\sqrt{\frac{i !(\lambda \mu)^{j-i}}{j ! r^{2(j-i)}}} \frac{\theta_{N-j}(s) \beta_{i}(s)}{|s I+B|} & , i<j \\ \frac{\theta_{N-i}(s) \beta_{i}(s)}{|s I+B|} & , i=j \\ \sqrt{\frac{j !(\lambda \mu)^{i-j}}{i ! r^{2(i-j)}}} \frac{\theta_{N-i}(s) \beta_{j}(s)}{|s I+B|} & , i>j\end{cases}
$$

From Eq. (19), we see that:

$$
\begin{aligned}
& \mathbb{F}(s)=A(s)^{-1} \mathbb{F}(0) \\
& \quad=\Phi^{-1}(s I+B)^{-1} \Phi \mathbb{F}(0) \\
& F_{n}{ }^{*}(s)=\sum_{k=0}^{N} \phi_{n}{ }^{-1} \phi_{k} c_{n k} F_{k}(0) \\
& =\phi_{n}{ }^{-1} \phi_{0} c_{n 0} F_{0}(0)
\end{aligned}
$$

where

$$
\phi_{n}{ }^{-1} \phi_{0}=\left\{\begin{array}{lc}
1 & , n=0 \\
\sqrt{\frac{r_{0}}{n ! r}\left(\frac{\lambda}{\mu}\right)^{n}} & , n=1,2, \ldots, N
\end{array}\right.
$$




$$
c_{n 0}= \begin{cases}\frac{\theta_{N}(s) \beta_{0}(s)}{|s I+B|} & , n=0 \\ \sqrt{\frac{(\lambda \mu)^{n}}{n ! r_{0} r^{2 n-1}}} \frac{\theta_{N}(s) \beta_{0}(s)}{|s I+B|}, & n=1,2, \ldots, N\end{cases}
$$

Let $\xi_{m}(m=0,1, \ldots, N)$ are the roots of polynomial of $|A(s)|$. These roots are the negative eigenvalues of the matrix $M(0)$. Since the matrix $M$ is positive definite, real and symmetric, the eigenvalues of Mare real, distinct, and positive. Hence the roots of $|A(s)|$ are real, distinct, and negative. The determinant $|s I+B|=s|M(s)|$ is equal to $s \prod_{m=1}^{N}\left(s-\xi_{m}\right)$, where $M(s)$ is given by:

$$
\begin{aligned}
& M(s)=\left(\begin{array}{cccccc}
s+\frac{\lambda_{0}}{r_{0}}+\frac{\mu}{r} & -\sqrt{\frac{\lambda \mu}{2 r^{2}}} & 0 & 0 \ldots & 0 & 0 \\
-\sqrt{\frac{\lambda \mu}{2 r^{2}}} & s+\frac{\lambda}{2 r}+\frac{\mu}{r} & -\sqrt{\frac{\lambda \mu}{3 r^{2}}} & 0 \ldots & 0 & 0 \\
0 & -\sqrt{\frac{\lambda \mu}{3 r^{2}}} & s+\frac{\lambda}{3 r}+\frac{\mu}{r} & \sqrt{\frac{\lambda \mu}{4 r^{2}}} \ldots & 0 & 0 \\
\cdot & \cdot & \cdot & 0 & 0 \\
\cdot & \cdot & \cdot & 0 & 0 \\
\cdot & \cdot & 0 & 0 \ldots & s+\frac{\lambda}{(N-2) r}+\frac{\mu}{r} & -\sqrt{\frac{\lambda \mu}{(N-1) r^{2}}} \\
0 & 0 & 0 & 0 \ldots & -\sqrt{\frac{\lambda \mu}{(N-1) r^{2}}} & s+\frac{\lambda}{(N-1) r}+\frac{\mu}{r}
\end{array}\right) \\
& F_{n}{ }^{*}(s)=\left\{\begin{array}{lc}
\frac{\theta_{N}(s) \beta_{0}(s) d_{o}}{|s I+B|} & , \quad n=0 \\
\sqrt{\frac{r_{0}}{n ! r}\left(\frac{\lambda}{\mu}\right)^{n}} \sqrt{\frac{(\lambda \mu)^{n}}{r_{0} r^{2 n-1} n !}} \frac{\theta_{N-n}(s) \beta_{0}(s) d_{0}}{|s I+B|} & , n=1,2, \ldots, N
\end{array}\right.
\end{aligned}
$$

or

$$
\begin{aligned}
& F_{n}^{*}(s)= \begin{cases}\frac{\theta_{N}(s) d_{o}}{s \prod_{i=1}^{N}\left(s-\xi_{i}\right)} & , \quad n=0 \\
\frac{1}{n !}\left(\frac{\lambda}{r}\right)^{n} \frac{\theta_{N-n}(s) d_{0}}{s \prod_{i=1}^{N}\left(s-\xi_{i}\right)} & , n=1,2, \ldots, N\end{cases} \\
& F_{n}^{*}(s)=\frac{p_{n}}{s}+\sum_{k=1}^{N} \frac{d_{n, k}}{s-\xi_{k}}
\end{aligned}
$$

where 


$$
d_{n, k}=\left\{\begin{array}{l}
\frac{\theta_{N}\left(\xi_{k}\right) d_{o}}{\xi_{k} \prod_{i=1, i \neq k}^{N}\left(\xi_{k}-\xi_{i}\right)}, \quad n=0 \\
\frac{1}{n !}\left(\frac{\lambda}{r}\right)^{n} \frac{\theta_{N-n}\left(\xi_{k}\right) d_{0}}{\xi_{k} \prod_{i=1, i \neq k}^{N}\left(\xi_{k}-\xi_{i}\right)}, n=1,2, \ldots, N
\end{array}\right.
$$

Applying Laplace inverse transform on Eq. (33), we get

$$
F_{n}(z)=p_{n}+\sum_{k=1}^{N} d_{n, k} e^{-\xi_{k} z}, n=0,1, \ldots, N ; z \geq 0
$$

Similarly, the closed form expressions for $F_{n}(z)$ of both models as given by (34) and (35) are obtained analytically. Therefore, the stationary distribution of the buffer content is given as follows:

$$
F(z)=\lim _{t \rightarrow \infty} \operatorname{Pr}(Z(t) \leq z)=\sum_{j=o}^{N} F_{j}(z)
$$

Or

$$
F(z)=1+\sum_{j=0}^{N} \sum_{k=1}^{N} d_{j, k} e^{-\xi_{k} z}
$$

Finally, the constants $p_{n}$ must satisfy the conditions (14). Also,

$$
p_{n}=\lim _{s \rightarrow 0} s F_{n}{ }^{*}(s), \text { and } \theta_{n}(0)=\left(\frac{\mu}{r}\right)^{n}
$$

Then,

$$
\begin{aligned}
& p_{n}=\frac{\rho^{n}}{n !} p_{0}, n=0,1, \ldots, N \\
& p_{0}=\left[1+\sum_{n=1}^{N} \frac{\rho^{n}}{n !}\right]^{-1}
\end{aligned}
$$

Where $\rho=\frac{\lambda}{\mu}$.

\section{Some performance measures in fluid model}

In this section, some crucial performance measures are considered. The formulation for these measures is given as follows:

\section{Server utilization}

The probability that buffer is non-empty is found by.

$$
\text { Utilization }=1-\sum_{j=0}^{N} F_{j}(0)=1-F_{0}(0) .
$$




$$
\text { Utilization }=1-d_{0}, 0<d_{0}<1
$$

where

$$
d_{0}=\frac{d}{r_{0}}=\frac{\left(r_{0}-r\right) p_{0}+r}{r_{0}}
$$

\section{Expected buffer content}

The expected buffer content $E(Z)$ can be written as:

$$
E(Z)=\int_{0}^{\infty}\left[1-\sum_{j=0}^{N} F_{j}(z)\right] d z .
$$

or

$$
E(Z)=\sum_{j=0}^{N} \sum_{k=1}^{N} \frac{d_{j, k}}{\xi_{k}}
$$

\section{Numerical illustrations}

This part illustrates the variations of the stationary distribution of the buffer content and the expected buffer content for varying values of parameters. Figure 1 depicts the behavior of the buffer content distribution, $F(z)$ against the buffer size $z$ for $\lambda=1, \mu=2$, $r_{0}=-1$, and $r=1$, for different value of $N$. Figure 2 presents the corresponding behavior of the expected buffer content against $\lambda$ for the same set of parameter values and $N=10$.

\section{Conclusions}

In this paper, a fluid queue model driven by an $M / M / 1 / N$ queue with discouraged arrivals is investigated. The steady-state distribution of the buffer occupancy is derived in terms of determinants of the top left and bottom right matrices using a computable matrix technique. As illustrated in Fig. 1, $F(z)$ is an increasing function, when the limit

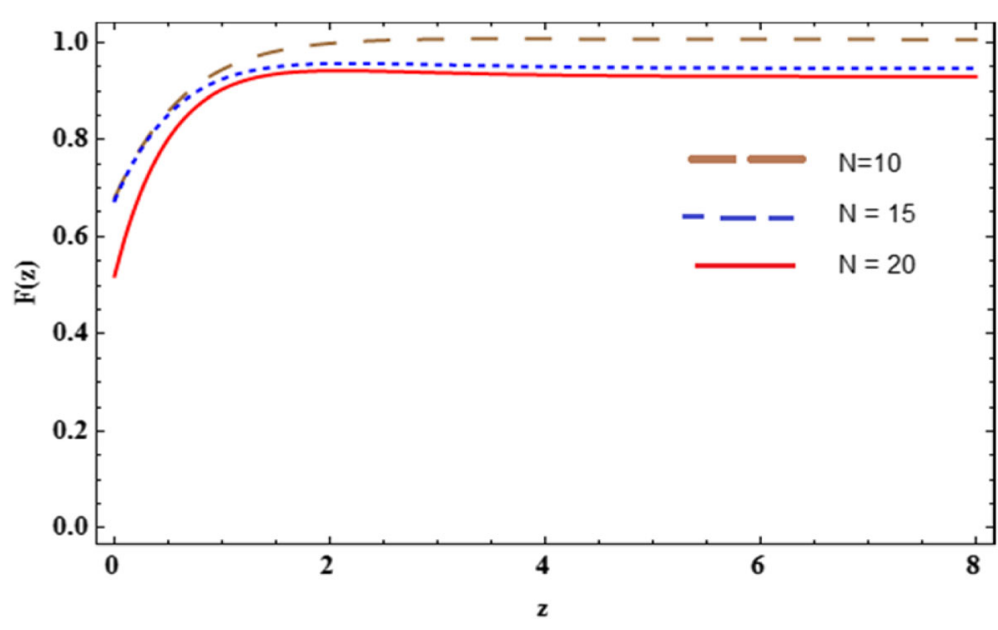

Fig. 1 The buffer content distribution, $F(z)$ vs. the buffer size $z$ for different values of $N$ 


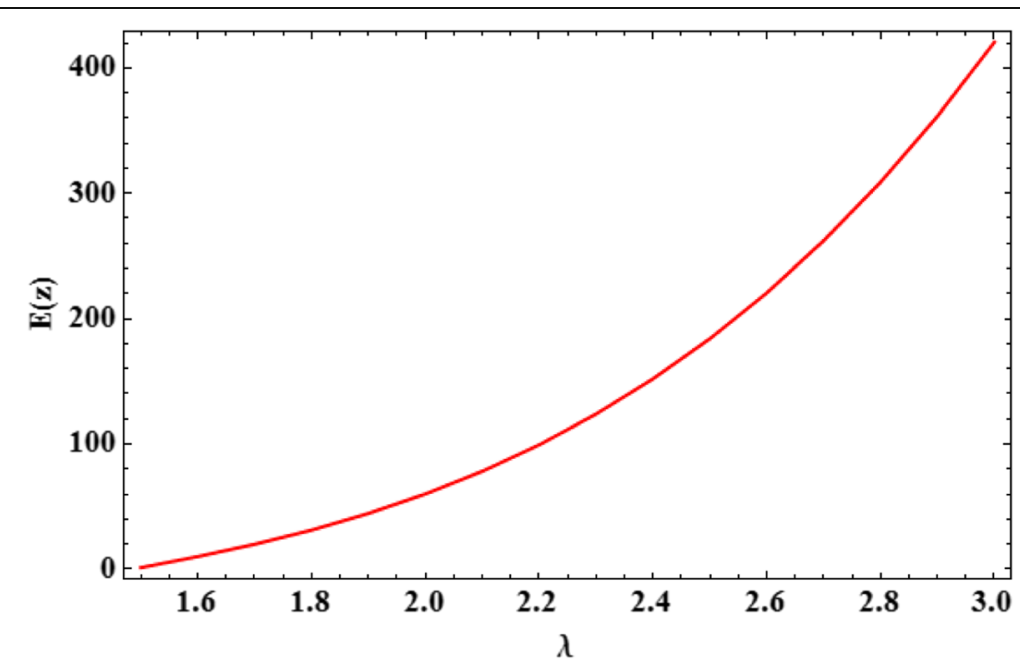

Fig. 2 The expected buffer content $E(z)$ against $\lambda$

waiting space is increased the distribution of the buffer content decrease with Nand the cumulative distribution function of buffer occupancy it is observed that there is a positive mass at $z \rightarrow 0$ and $F(z) \rightarrow 1$ as $z \rightarrow \infty$. Hence, this means that the buffer occupancy has mixed distribution. Also, Fig. 2 shows the mean of the stationary buffer content with arrival rate $\lambda$. Finally, some performance measures such as server utilization and mean buffer content are acquired.

Authors' contributions

The authors contributed equally to this work. All authors read and approved the final manuscript.

Funding

Not applicable

Availability of data and materials

Not applicable

\section{Competing interests}

The authors declare that they have no competing interests.

\section{Author details}

'Department of Statistics, Faculty of Commerce, Al-Azhar University, Girls' Branch, Dakhlia, Egypt. ²Department of Mathematics, College of Sciences and Arts in Ar-Rass, Qassim University, Ar-Rass, Saudi Arabia. ${ }^{3}$ Department of Mathematics and Statistics, Faculty of Management Technology and Information Systems, Port Said University, Port Said, Egypt.

Received: 31 October 2019 Accepted: 10 February 2020

Published online: 25 March 2020

\section{References}

1. Adan, I., Resing, J.: Simple analysis of a fluid queue driven by an M/M/1 queue. Queueing Systems. 22, 171-174 (1996). https://doi.org/10.1007/bf01159399

2. Anick, D., Mitra, D., Sondhi, M.: Stochastic theory of a data-handling system with multiple sources. Bell Syst. Tech. J. 61, 1871-1894 (1982). https://doi.org/10.1002/j.1538-7305.1982.tb03089.x

3. Barbot, N., Sericola, B.: Stationary solution to the fluid queue fed by an M/M/1 queue. J. Appl. Probab. 39, 359-369 (2002). https://doi.org/10.1239/jap/1025131431

4. Kulkarni, V.G.: Fluid models for single buffer systems. Frontiers in queueing: Models and applications in science and engineering, pp. 321-338 (1997)

5. Mitra, D.: Stochastic theory of a fluid model of producers and consumers coupled by a buffer. Advances Applied Probability. 20, 646-676 (1988)

6. Lenin, R.B., Parthasarathy, P.R.: Fluid queues driven by an M/M/1/N queue. Math Problems in Eng. 6, 439-460 (2000). https://doi.org/10.1155/S1024123X00001423

7. Lenin, R.B., Parthasarathy, P.R.: A computational approach for fluid queues driven by truncated birth-death processes. Methodol. Comput. Appl. Probab. 9, 373-392 (2000). https://doi.org/10.1023/A:1010010201531 
8. Mao, B., Wang, F., Tian, N.: Fluid model driven by an $M / M / 1 / N$ queue with multiple exponential vacation. J Comput Inf Syst. 6, 1809-1816 (2010)

9. Mao, B., Wang, F., Tian, N.: Fluid model driven by an $M / M / 1 / N$ queue with single exponential vacation. Int. J. Inf. Manag. Sci. 21, 29-40 (2010)

10. Viswanathan, A., Vandana, G., Dharmaraja, S.: A fluid queue modulate by two independent birth-death processes. Comput. Math. Appl. 60, 2433-2444 (2010). https://doi.org/10.1016/j.camwa.2010.08.039

11. Guillemin, F., Sericola, B.: Volume and duration of losses in finite buffer fluid queues. J. Appl. Probab. 52, 826-840 (2015). https://doi.org/10.1239/jap/1445543849

12. Vijayashree, K.V., Anjuka, A.: Stationary analysis of a fluid queue driven by an $M / M / 1 / N$ queue with disaster and subsequent repair. Int. J. Operational Research. 31, 461-477 (2018). https://doi.org/10.1504/JOR.2018.090427

13. Bruno, S.: Markov Chains: Theory, Algorithms and Applications. ISTE Series, Wiley (2013). Print ISBN: 9781848214934 Online ISBN: 9781118731543. https://doi.org/10.1002/9781118731543

14. Vijayalakshmi, T., Thangaraj, V: Transient analysis of a fluid queue driven by a chain sequenced birth and death process with catastrophes. Int. J. Math. Oper. Res. 8, 164-184 (2016). https://doi.org/10.1504/JMOR.2016.074853

15. Soares, A., Latouche, G.: Matrix-analytic methods for fluid queues with finite buffers. Perform. Eval. 63, 295-314 (2006). https://doi.org/10.1016/.jpeva.2005.02.002

\section{Publisher's Note}

Springer Nature remains neutral with regard to jurisdictional claims in published maps and institutional affiliations.

Submit your manuscript to a SpringerOpen ${ }^{\circ}$ journal and benefit from:

- Convenient online submission

Rigorous peer review

- Open access: articles freely available online

High visibility within the field

- Retaining the copyright to your article

Submit your next manuscript at $\boldsymbol{\nabla}$ springeropen.com 